\title{
Distribution of Schmallenberg Virus and Seroprevalence in Belgian Sheep and Goats
}

\author{
E. Méroc ${ }^{1, *}$, N. De Regge ${ }^{2, *}$, F. Riocreux ${ }^{1}$, A. B. Caij ${ }^{2}$, T. van den Berg ${ }^{2}$ and Y. van der Stede ${ }^{1,3}$ \\ 1 Veterinary and Agrochemical Research Centre, Coordination of Veterinary Diagnostics Epidemiology and Risk Analysis, Brussels, Belgium \\ 2 Veterinary and Agrochemical Research Centre, Operational Directorate Viral Diseases, Brussels, Belgium \\ 3 Laboratory of Immunology, Faculty of Veterinary Medicine, Ghent University, Merelbeke, Belgium
}

\section{Keywords:}

Schmallenberg virus; seroprevalence; spatial cluster; ELISA; sheep

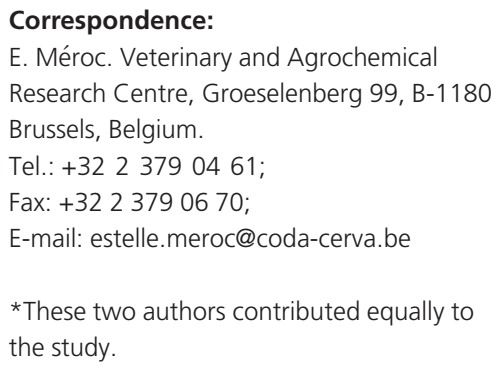

\begin{abstract}
Summary
A serological survey to detect Schmallenberg virus (SBV)-specific antibodies by ELISA was organized in the Belgian sheep population to study the seroprevalence at the end of the epidemic. One thousand eighty-two sheep samples which were collected from 83 herds all over Belgium between November 2011 and April 2012 were tested. The overall within-herd seroprevalence and the intraclass correlation coefficient were estimated at $84.31 \%$ (95\% CI: 84.19-84.43) and 0.34, respectively. The overall between-herd seroprevalence was $98.03 \%$ (95\% CI: 97.86-98.18). A spatial cluster analysis identified a cluster of six farms with significantly lower within-herd seroprevalence in the south of Belgium compared with the rest of the population $(P=0.04)$. It was shown that seroprevalence was associated to flock density and that the latter explained the presence of the spatial cluster. Additionally, 142 goat samples from eight different herds were tested for SBV-specific antibodies. The within-herd seroprevalence in goats was estimated at $40.68 \%$ (95\% CI: $23.57-60.4 \%)$. The results of the current study provided evidence that almost every Belgian sheep herd has been in contact with SBV during 2011 and should be taken into consideration as part of comprehensive SBV surveillance and control strategies.
\end{abstract}

\section{Introduction}

At the end of summer 2011, an unidentified disease appeared in adult cattle in several farms located in Germany and the Netherlands (ProMED-Mail, 2011b). The animals showed non-specific acute symptoms [milk drop, watery diarrhoea and hyperthermia $\left(>40^{\circ} \mathrm{C}\right)$ ] and recovered after a few days. The Friedrich Loeffler Institute (FLI, Germany) conducted a metagenomic analysis in cattle blood samples and identified a new virus phylogenetically closely related to viruses of the Simbu serogroup of the genus Orthobunyavirus of the family Bunyaviridae (Hoffmann et al., 2012). This virus was shown to be responsible for the above-mentioned symptoms and was named after the place in Germany it was first identified: the Schmallenberg virus (SBV; Hoffmann et al., 2012; Muskens et al., 2012). Several viruses from the Simbu serogroup such as
Shamonda, Akabane and Aino viruses are known to be teratogenic for ruminants when infected during a vulnerable period during gestation and may lead to abortion or congenital malformation (Yanase et al., 2012). In line with this, it was observed that starting from December 2011, cattle, sheep and goats in Germany and the Netherlands suffered from abortions and stillborn malformed offspring with signs including hydranencephaly and arthrogryposis. Realtime reverse transcription PCR (RT-qPCR) was made available by the FLI and was used to analyse SBV suspected animals (Hoffmann et al., 2012). Thus far, most SBV cases have been confirmed in Belgium, France, Germany, the Netherlands and the United Kingdom, and more sporadic cases were confirmed in Italy, Luxembourg, Spain, Switzerland, Denmark and Sweden (EFSA, 2012). In Belgium, the first reports of malformation in newborn lambs occurred mid-December 2011 and were confirmed by RT-qPCR in a 
farm from the North of the country located near the Dutch border (ProMED-Mail, 2011a). At the end of August 2012, SBV had been detected by RT-qPCR in 407 cattle holdings, 167 sheep holdings and two goat holdings in Belgium.

The viruses from the Simbu serogroup are all arthropodborne viruses mainly transmitted between animals via Culicoides spp. and Culex mosquitoes (WHO, 1961; Takahashi et al., 1968; Yanase et al., 2005). The detection of SBV in pools of heads of Culicoides obsoletus sensu stricto and Culicoides dewulfi captured in Belgium during the period September-October 2011 (ProMED-Mail, 2012; De Regge et al., 2012) suggests a role of Culicoides in the transmission of SBV. So far, only transplacental and arthropodborne transmissions have been described for SBV and there is no evidence that the virus could be transmitted horizontally (EFSA, 2012). Considering that SBV seems to be spread by Culicoides, it can be assumed that in Northern Europe it will be seasonally dependent and the important question at the moment is to know if a new wave of viral circulation could be expected in 2012 during the period of activity of Culicoides. Knowledge about the immunological anti-SBV status in the host population that has been acquired during the first circulation period in 2011 is needed to address this question as it could largely influence the outcome of a possible new appearance of the virus. Seroprevalence surveys in affected countries are therefore essential to estimate the real impact of SBV infection in 2011 and help to predict the potential further spread of the disease. In this context, a serological survey was conducted in the Belgian small ruminant population to investigate the situation at the end of the first wave of the SBV epidemics.

\section{Material and Methods}

\section{Sampling design}

The SBV sampling frame consisted of serum samples initially collected from sheep and goats in the context of the Maedi-Visna and Caprine Arthritis and Encephalitis Programme for trade certification (Royal Decree 24-03-1993). This programme works on a voluntary basis. After applying for the accreditation scheme, two samplings are carried out in the flock at a time interval of 6-12 months. All animals older than 1 year are sampled. All samples collected between 4 November 2011 and 4 April 2012 were included in this study.

\section{Diagnostic methods}

Blood samples were collected by farm veterinarians. Blood samples were conditioned, and serum was collected at the regional laboratories in Belgium: 'Dierengezondheidszorg Vlaanderen' and the 'Association Régionale de Santé et d'Identification Animales'. The serum samples were subsequently sent to the Veterinary and Agrochemical Research Centre (CODA-CERVA) where they were tested for the presence of SBV-specific antibodies using a commercially available ELISA kit (ID Screen ${ }^{\circledR}$ Schmallenberg virus Indirect ELISA kit, Montpellier, France). The validation of the test conducted by the manufacturer, using serum samples originating from France and Germany, demonstrated a relative sensitivity of $96.47 \%$ (95\% CI: 93.43-98.13\%) and specificity of $99.75 \%$ (95\% CI: 99.26-99.92\%; IDVET, 2012). Results were expressed as $S / P$ percentage using the optical densities (OD) from the ELISA reader (S/P $\left.\%=\mathrm{OD}_{\text {sample }} / \mathrm{OD}_{\text {positive control }} \times 100\right)$. A cut-off prescribed by the manufacturer was used to assign the samples into a category (positive, negative and doubtful). Samples that presented an S/P lower or equal to $60 \%$, between 60 and $70 \%$ and $>70 \%$ were, respectively, considered as negative, doubtful and positive. In this study, the doubtful results were considered as positive in the data analysis.

\section{Seroprevalence}

The sampling design of the study implied a hierarchical structure of the data, with animals typically clustered within flocks. Therefore, a marginal model, the generalized estimating equations (Liang and Zeger, 1986), which takes into account the resulting correlation among animals, was used to estimate the within-herd seroprevalence with 95\% confidence intervals. In this study, an exchangeable working correlation was assumed. The xtgee procedure in STATA $^{\circledR}$ software version 10.0 was applied to fit the model. The within-herd seroprevalence estimates of the positive sheep flocks were plotted as a density-scale histogram.

A generalized linear mixed model with normally distributed random intercepts for each flock $u_{i} \sim \operatorname{Normal}\left(0, \sigma^{2}\right.$; Molenberghs and Verbeke, 2005) was then used to establish the correlation between the infection status of two animals within a flock. The gllamm procedure was applied to estimate the intraclass correlation coefficient (ICC):

$$
\mathrm{ICC}=\frac{\sigma^{2}}{\sigma^{2}+\pi^{2} / 3}
$$

Between-herd seroprevalence (probability that a flock was infected) was estimated using a logistic regression model with the logit function. For the purpose of this study, a flock was considered as positive if at least one of the animals sampled was positive, otherwise it was considered negative.

Design effect was taken into account by weighting each observation by the inverse of the sampling probability (number of flocks sampled per province/number of flocks per province and number of animals sampled per flock/ number of animals per flock). 


\section{Spatial analyses}

To predict within-herd seroprevalence for unmeasured locations in Belgium, an interpolation from the data points was performed by the method of inverse distance weighting based on the six nearest neighbours (Shepard, 1968; ESRI, 1996). A map showing the distribution of within-herd seroprevalence estimates was produced using ARCGIS, version 9.3.1 (ESRI).

A purely spatial weighted normal model was used to scan for clusters of sampled flocks with either high or low levels of within-herd seroprevalence. In short, this is done by using a variable circular window size and noting the number of observed and expected observations inside the window at each location. The model tests the null hypothesis that seroprevalence is homogeneously distributed among the flocks (no clusters of flocks with unusually high or low seroprevalence). The method uses a likelihood ratio test to identify clusters. To test the significance of this likelihood, 1000 Monte Carlo simulations were performed to obtain its distribution, and clusters with $P$-value $<0.05$ were considered as statistically significant. Flock density by municipality was calculated using census data and farm $X$ and $Y$ coordinates extracted from the central identification and registration system of the Belgian Federal Agency for the Safety of the Food Chain (SANITEL). Subsequently, we conducted the spatial analysis adjusting for flock density to investigate for the existence of clusters after taking into account this potentially explanatory variable. To do so, the residuals obtained after fitting a univariable linear regression model with flock density as covariate were used as observed values instead as the original within-herd prevalence estimates. In addition, the Spearman's rank correlation coefficient was estimated to investigate the correlation between within-herd seroprevalence and flock density. All calculations were made using SATSCAN 8.2.1. (Kulldorff, 1997) and STATA ${ }^{\circledR}$ software version 10.0.

\section{Results}

\section{Seroprevalence}

A total of 1082 sheep from 83 flocks were sampled between 4 November 2011 and 4 April 2012. The number of sampled animals per flock ranged from 2 to 110 $($ median $=8)$. The geographical localization of the sampled flocks is shown in Fig. 1. The within-herd seroprevalence and the ICC in sheep were estimated at $84.31 \%$ (95\% CI: 84.19-84.43) and 0.34, respectively. In the seropositive flocks (at least one animal detected seropositive), the predicted value for within-herd seroprevalence ranged from $38.6 \%$ to $96.71 \%$ (median $=89.28 \%$; Fig. 2 ). The between-herd seroprevalence in sheep was $98.03 \%(95 \%$ CI: 97.86-98.18).

In addition, 142 goats from eight flocks were also sampled during the same period (Fig. 1). The within-herd seroprevalence was estimated at $40.68 \%$ (95\% CI: $23.57-$ $60.4 \%$ ) for goats.

\section{Spatial analyses}

Figure 3 illustrates the geographical distribution of withinherd seroprevalence with interpolated predictions for

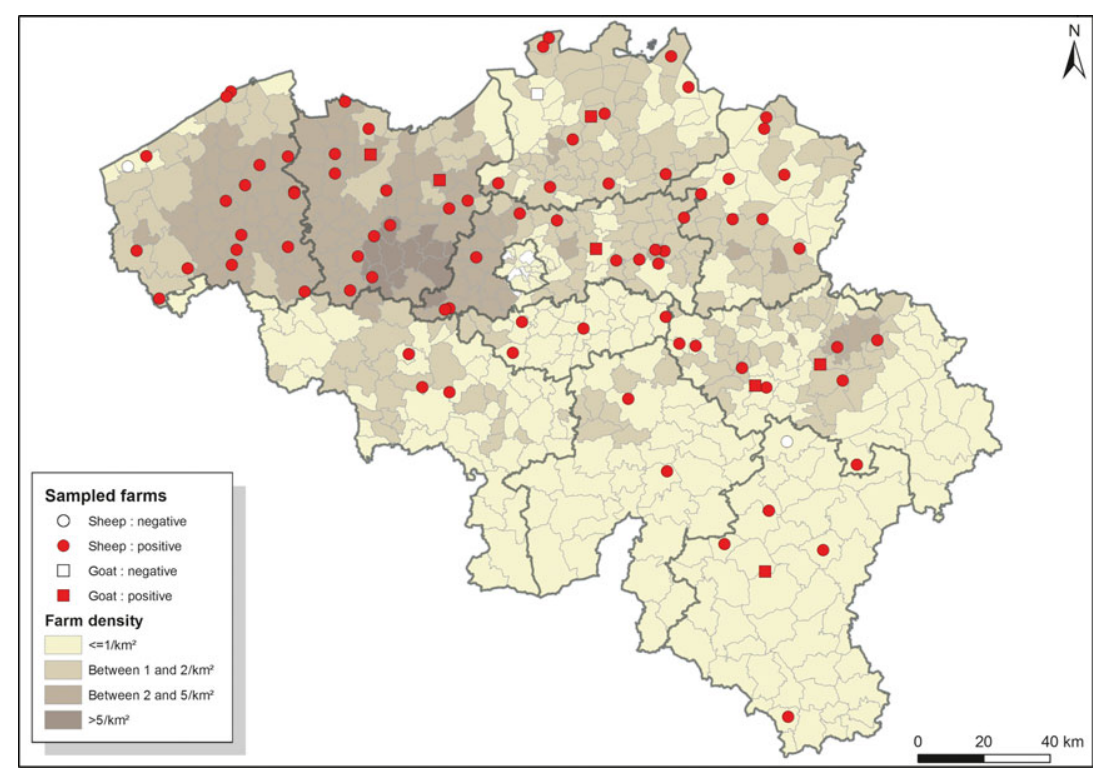

Fig. 1. Sheep and goat flocks sampled for Schmallenberg virus (SBV) in Belgium between 4 November 2011 and 4 April 2012 and flock density (sheep and goat) at the municipality level. 


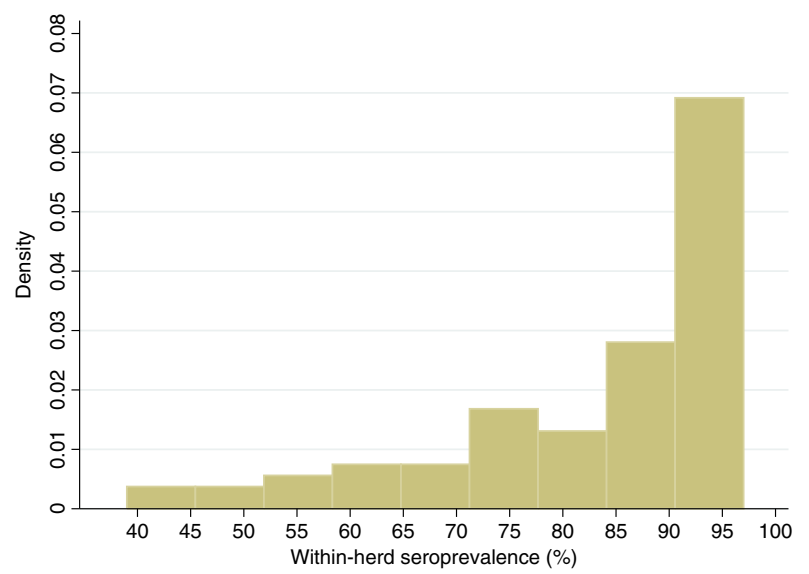

Fig. 2. Density-scale histogram of within-herd seroprevalence estimates (\%) in Belgian sheep flocks positive to Schmallenberg virus (SBV).

unmeasured points. The prevalence is globally high (80$90 \%$ ) and evenly distributed across the country. The regions where seroprevalence was the highest were those located in the north-west of Belgium. The levels of withinherd seroprevalence were low in the south-eastern part of the country, and a significant spatial cluster of six farms was identified in that region. The mean seroprevalence in this cluster was $59.5 \%$ and outside the cluster $84.87 \%$ $(P=0.04$; Fig. 3). Another spatial cluster of $32 \mathrm{farms}$ in the western part of Belgium had, on the other hand, a higher mean seroprevalence observed $(87.94 \%)$ than expected $(79.86 \%)$, but was not significant $(P=0.08)$. Ovine flock density at the municipality level ranges from 0 to 8 flocks $/ \mathrm{km}^{2}$. The spatial cluster scanning was conducted again, taking flock density into account, and no significant cluster was detected. A statistically significant Spearman's rho of $0.25(P=0.02)$ was obtained, indicating a moderate positive association between within-herd seroprevalence and flock density.

\section{Discussion}

The results of this survey demonstrated that the SBV seroprevalence in sheep was extremely high in winter 20112012. Schmallenberg virus seems to have circulated all over Belgium during the 2011 vector season as the between-herd seroprevalence was almost $100 \%$. The interpolation of within-herd seroprevalence estimates showed a high level of infection all over the territory. Within the positive flocks, seroprevalence ranged from $38.6 \%$ to $96.71 \%$, but the great majority of the flocks (70\%) had a seroprevalence above $80 \%$. An ICC of 0.34 was found, indicating that the correlation between two animals within a flock with respect to SBV result was high. The ICC of a livestock infectious disease is usually $<0.2$ and ranges from 0.04 to 0.42 (Otte and
Gumm, 1997). From a screening among Dutch dairy cattle by virus neutralization, a seroprevalence on animal level of $72.5 \%$ was found (Elbers et al., 2012). The first preliminary results of studies from other affected countries also showed a high seroprevalence with results from Germany and France at animal level ranging from 61 to $100 \%$ in cattle (EFSA, 2012).

The regions where seroprevalence was the highest were those located in the north-east of Belgium corresponding to areas with high sheep density. Indeed, the results of this study demonstrated that the geographical pattern of seroprevalence was dependent on that factor. The existence of a spatial cluster with low seroprevalence seems to be linked to the fact that flock density in this part of the country is very low and may result in a smaller virus reservoir in the environment and, consequently, a weaker risk to disseminate the infectious disease by the vector, compared with high-density regions. It should be noticed that the predicted seroprevalence in the south of Belgium results from an interpolation of sparser data compared with other areas, and this will eventually have an impact on the confidence intervals of the predictions.

The sampling was performed on a voluntary basis, and the interested owners were those who practise trade. Therefore, the flocks that were sampled may have had some particular features in their management, size, etc. This type of sampling obviously presents opportunity risk of selection bias, which must be accounted for when considering the results of the study. It seems also important to mention that sample size in the screening was limited to available samples and that a lack in number of observations will inevitably lead to less precise estimations. However, pertaining to sheep, the number of animals sampled per flock seems reasonable, given that the median size of Belgian flocks is 4 and that a median number of eight animals were sampled. At the flock level, expecting a high between-herd seroprevalence (90-95\%), a sample size of 73-139 flocks would be required to reach a desired confidence level of $95 \%$ and accepted error of 5\%. Thus, the 1082 sheep from 83 flocks analysed in this study seem suitable to give reasonable estimates of seroprevalence in Belgium. Moreover, representativeness is obtained as the sampled flocks were well distributed within the 10 Belgian provinces, and the sampling probability of each animal and farm was taken into account in the analysis by weighting observed values.

The current screening was not able to give very precise estimates for the goat population, as pointed out by the considerably large confidence interval, but gives an indication that the infection level in this species might be lower than it is in sheep and cattle. This would mean that a considerable portion of the goat population remains susceptible and may be sufficient to maintain the infection in Belgium during the following transmission season. 


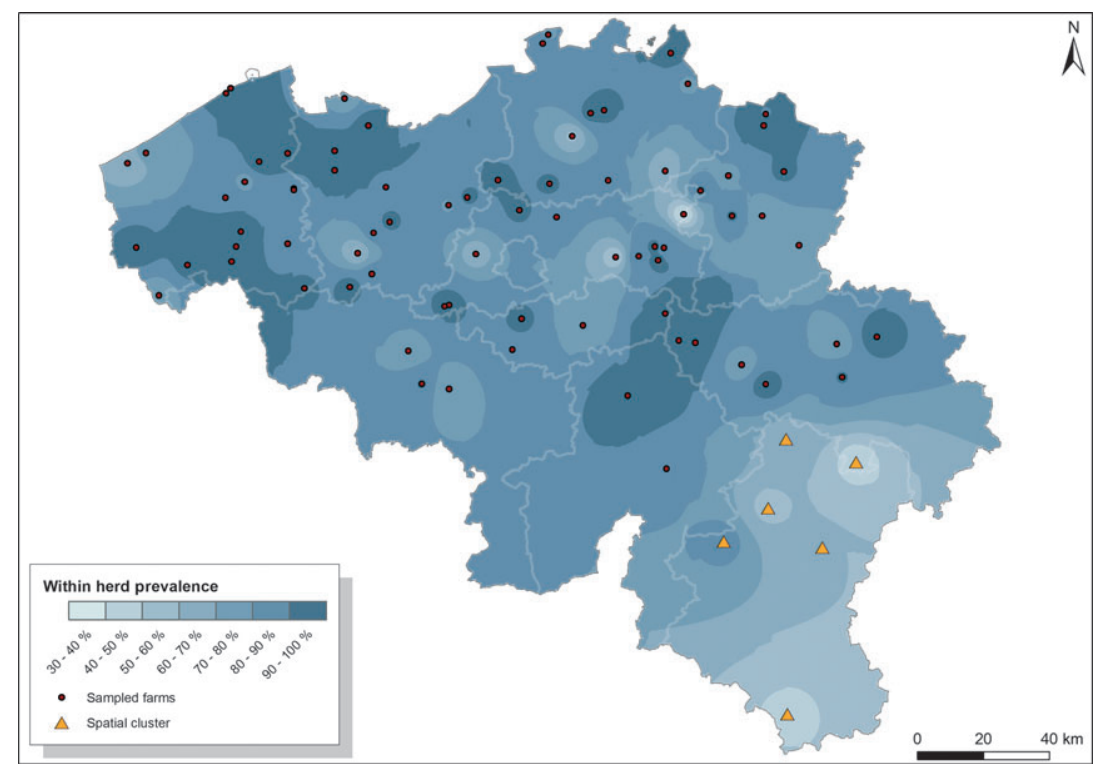

Fig. 3. Geographical distribution of Schmallenberg virus (SBV) within-herd seroprevalence in Belgian sheep flocks and spatial cluster for low withinherd seroprevalence.

Our results show that there is a high discrepancy between the number of flocks in which the presence of SBV was confirmed by rRT-PCR [167 (0.6\% of the total flock population in Belgium)] and the number of flocks that have been infected with the virus based on the serological results. The first factor to explain this difference could be underreporting. This can be expected when dealing with a new virus for which farmers and animal health professionals are completely unfamiliar. Moreover, SBV is not a notifiable disease in Belgium, and there is no obligation to report infections to the authorities. The second factor is related to the absence of symptoms in adult sheep. Thus far, the disease has only been observed in lambs after vertical transmission. Indeed, if the ewe was contaminated before or after the vulnerable period during gestation, the infection would have passed unnoticed. The exact vulnerable period in sheep is currently being investigated, but in reference to other viruses of the Simbu serogroup, should last more or less 3 weeks (Hashinguchi et al., 1979; Steukers et al., 2012). Finally, a third factor is the absence of virus in some lambs despite clinical suspicion indicating a possible clearance of the virus before birth.

It is interesting to note that despite a shorter viraemia (Hoffmann et al., 2012), SBV has spread much faster than bluetongue virus serotype 8 (BTV-8) did, after its emergence in the same region 5 years ago (Elbers et al., 2008; Méroc et al., 2008). Several possibilities can be suggested to try and explain this difference of dissemination between the two Culicoides-transmitted viruses: (i) additional routes of transmission exist for SBV, (ii) the host range of SBV is not limited to ruminants, (iii) the infectious dose and the competence of Culicoides for transmitting the two viruses are not similar (Kirkland, 2012), (iv) Schmallenberg virus was already present in Belgium prior to 2011, and (v) no legal measures such as live animal standstill were taken for $\mathrm{SBV}$ as it was the case for BTV-8. Although some of those hypotheses have already been investigated, more research is still necessary to elucidate clearly which of those should be retained.

In conclusion, the results of this study provided evidence that almost every Belgian sheep herd has been at one point in contact with SBV. Thus, if we extrapolate from other viruses from the Simbu group like the Akabane virus, which is the most well-known species of the serogroup, this means that the vast majority of sheep could by now have acquired natural protective immunity against SBV which may last for a few years (Anonymous, 2008; Steukers et al., 2012). Therefore, it seems probable that even if the virus overwinters, regions with high seroprevalence such as Belgium should not experience a similar epidemic in 20122013 and that only animals located in areas that are still free of SBV could in turn become infected, just as lambs after the waning of maternally derived antibody. Regions neighbouring affected areas are probably the most at risk for virus circulation, especially as Culicoides from the obsoletus group, the identified vectors of SBV are present in most European countries except northern parts of Norway and Sweden (EFSA, 2012). Nevertheless, even in highly immunized livestock, because of all newborn animals, part of the host population will continuously remain susceptible. For these different reasons, the cluster identified in the south of Belgium with lower seroprevalence than the rest of the 
country should be prioritized in the context of future surveillance actions. In Australia, Akabane disease is controlled by acting on herd management, for instance by moving susceptible animals into endemic areas in time to develop immunity before they are first bred (Kirkland, 2012).

The findings presented in this study should be taken into consideration as part of comprehensive SBV surveillance strategy. It is important to underline the paramount need to answer specific questions such as the duration of postinfection protective immunity and the exact host range, especially, as the emergence of exotic and newly identified arthropod-borne pathogens seems to happen more and more frequently in recent years.

\section{Acknowledgements}

The authors gratefully acknowledge Sylvie Malbrecq, the staff of ARSIA and DGZ for providing the samples and the administrative data, as well as the technical assistance of Laura Georges and Muriel Verhoeven. The authors thank the veterinarians and all the persons who have contributed to the collection of data for their cooperation in this study.

\section{References}

Anonymous 2008: Bunyaviral diseases of animals. OIE Terrestrial Manual 2008, 1166-1163.

De Regge, N., I. Deblauwe, R. De Deken, P. Vantieghem, M. Madder, D. Geysen, F. Smeets, B. Losson, T. van den Berg, and A. B. Cay, 2012: Detection of Schmallenberg virus in different Culicoides spp. by real time RT-PCR. Transbound. Emerg. Dis. 59, 471-475.

European Food Safety Authority, EFSA 2012. 'Schmallenberg' virus: analysis of the epidemiological data and impact assessment. Available at: http://www.efsa.europa.eu/fr/efsajournal/ pub/2768.htm (accessed July 1, 2012).

Elbers, A. R., J. Popma, S. Oosterwolde, P. A. van Rijn, P. Vellema, and E. M. A. van Rooij, 2008: A cross-sectional study to determine the seroprevalence of bluetongue virus serotype 8 in sheep and goats in 2006 and 2007 in the Netherlands. BMC Vet. Res. 4, 33.

Elbers, A. R., W. L. Loeffen, S. Quak, E. de Boer-Luijtze, A. N. van der Spek, R. Bouwstra, R. Maas, M. A. Spierenburg, E. P. de Kluijver, G. van Schaik and W. H. van der Poel, 2012: Seroprevalence of Schmallenberg virus antibodies among dairy cattle, the Netherlands, winter 2011-2012. Emerg. Infect. Dis. 18, 1065-1071. doi:10.3201/eid1807.120323.

ESRI 1996: Arc View Spatial Analyst. Arcview Spatial Analysis Using Raster and Vector Data. Environmental Systems Research Institute Inc., Redlands, CA, 81-11.

Hashinguchi, Y., K. Nanba and T. Kumagai, 1979: Congenital abnormalities in newborn lambs following Akabane virus infection in pregnant ewes. Natl Inst. Anim. Health Q. (Tokyo) $19,1-11$.
Hoffmann, B., M. Scheuch, D. Höper, R. Jungblut, M. Holsteg and H. Schirrmeier, 2012: Novel orthobunyavirus in cattle, Europe, 2011. Emerg. Infect. Dis. 2012, 469-472.

IDVET 2012: Validation report, ID screen Schmallenberg virus indirect. 06/04/2012.

Kirkland, P. D., 2012: Akabane and other Simbu viruses: epidemiology, pathogenesis and impact. Accessed on 3 July 2012. Available at: http://ec.europa.eu/food/animal/diseases/ schmallenberg_virus/docs/akabane_other_sbv_en.pdf (accessed July 9, 2012).

Kulldorff, M., 1997: A spatial scan statistic. Communications in Statistics. Theory Methods 26, 1481-1496.

Liang, K. Y. and S. L. Zeger, 1986: Longitudinal data analysis using generalized linear models. Biometrika 73, 13-22.

Méroc, E., C. Faes, C. Herr, C. Staubach, B. Verheyden, T. Vanbinst, F. Vandenbussche, J. Hooyberghs, M. Aerts, K. De Clercq and K. Mintiens, 2008: Establishing the spread of bluetongue virus at the end of the 2006 epidemic in Belgium. Vet. Microbiol. 131, 133-144.

Molenberghs, G. and G. Verbeke, 2005: Models for Discrete Longitudinal Data. Springer Series in Statistics. Springer, New York.

Muskens, J., A. J. Smolenaars, W. H. van der Poel, M. H. Mars, L. van Wuijckhuise, M. Holzhauer, H. van Weering and P. Kock, 2012: Diarrhea and loss of production on Dutch dairy farms caused by the Schmallenberg virus. Tijdschr. Diergeneeskd. 137, 112-115.

Otte, M. J. and I. D. Gumm, 1997: Intracluster correlation coefficient of 20 infections calculated from the results of cluster-sample surveys. Prev. Vet. Med. 31, 147-150.

ProMED-Mail 2011a. Schmallenberg virus - Europe (07): (Belgium) congenital malformations, ovine. ProMED-mail 2011; 23 Dec: 20111223.3665. Available at http://www.promedmail.org (accessed July 2, 2012).

ProMED-Mail 2012. Schmallenberg virus - Europe (26): (Belgium, Germany) vector, morphology. ProMED-mail 2011; 11 March: 20120311.1066949. Available at http://www. promedmail.org (accessed July 2, 2012).

ProMED-Mail 2011b. Undiagnosed illness, bovine-Germany, Netherlands (02): new virus suspected. ProMED-mail 2011; 19 Nov: 20111119.3404. Available at http://www.promedmail. org (accessed July 10, 2012).

Shepard, D., 1968: A two-dimensional interpolation function for irregularity-spaced data. Proceedings of the 23rd National Conference ACM, New York, NY, USA, 517-524.

Steukers, L., G. Bertels, A. B. Cay and H. J. Nauwynck, 2012: Schmallenberg virus: emergence of an Orthobunyavirus among ruminants in Western Europe. Vlaams. Diergeneeskd. Tijdschr 81, 119-126.

Takahashi, K., A. Oya, T. Okazda, R. Matsuo and M. Kuma, 1968: Aino virus, a new member of Simbu group of arbovirus from mosquitoes in Japan. Jpn. J. Med. Sci. Biol. 21, 95-101.

World Health Organization, WHO, 1961: Arthropod-borne viruses, report of a study group; [Geneva, 5-10 September 1960]. World Health Organization Technical Report Series, 219, 68 pp. 
Yanase, T., K. Maeda, T. Kato, S. Nyuta, H. Kamata and M. Yamakawa, 2005: The resurgence of Shamonda virus, an African Simbu group virus of the genus Orthobunyavirus, in Japan. Arch. Virol. 150, 361-369.

Yanase, T., T. Kato, M. Aizawa, Y. Shuto, H. Shirafuji, M. Yamakawa and T. Tsuda, 2012: Genetic reassortment between
Sathuperi and Shamonda viruses of the genus Orthobunyavirus in nature: implications for their genetic relationship to Schmallenberg virus. Arch. Virol. 157, 1611-1616. 